\title{
Angiolymphoide Hyperplasie mit Eosinophilie
}

\author{
Dj. Djawari ${ }^{1}$ \\ H. J. Rumpelt ${ }^{2}$
}

\section{Angiolymphoid Hyperplasia with Eosinophilia}

\section{Zusammenfassung}

Eine 49-jährige Patientin zeigte einen $1,5 \mathrm{~cm}$ durchmessenden, derben und livid verfärbten Nodus kutan-subkutan gelegen temporoparietal rechts, der angeblich seit einem Jahr symptomlos bestand und langsam wuchs. Nach der In-toto-Exzision des Tumors wurde histologisch die Diagnose einer Angiolymphoiden Hyperplasie mit Eosinophilie gestellt.

\section{Einleitung}

Die Angiolymphoide Hyperplasie mit Eosinophilie (AHE) stellt eine seltene vaskuläre entzündliche Erkrankung unbekannter Ätiologie dar [7]. Klinisch zeigen sich meist einzelne angiomartige Knoten bzw. Papeln, mit bevorzugter Lokalisation an Kopf, Hals und Nacken, insbesondere im Bereich der Ohren [12]. Histologisch ist die AHE durch eine umschriebene, dermal bis teils subkutan gelegene, Gefäßproliferation kleiner atypischer Blutgefäße, umgeben von einem ausgeprägten Infiltrat aus eosinophilen Granulozyten, Histiozyten und Lymphozyten, gekennzeichnet [5]. In ihrem Verlauf handelt es sich um eine chronisch progrediente Erkrankung, ohne maligne Transformation. Da jedoch Rezidivneigung besteht, sollte ein operatives Vorgehen anderen Therapieverfahren vorgezogen und der Tumor jeweils in sano exzidiert werden.

\section{Abstract}

A 49-years-old woman showed a slowly growing tumor on her head temporoparietally at the right side. The cutan subcutan localized nodus had a $1.5 \mathrm{~cm}$ diameter without any symptoms. After the in toto excision of the tumor an angiolymphoid hyperplasia with eosinophilia was found histologically. The patient showed no recidiv until now, after 7 years.

\section{Fallbericht}

\section{Anamnese}

Die 49-jährige Patientin berichtete über einen seit zirka einem Jahr langsam wachsenden Knoten temporo-parietal rechts. Subjektive Beschwerden wurden nicht angegeben. Andere Erkrankungen lagen nicht vor.

\section{Befund}

Es fand sich ein $1,5 \mathrm{~cm}$ durchmessender, halbkugelig imponierender, scharf begrenzter, derber, livid verfärbter und kutan-subkutan gelegener Nodus temporoparietal rechts mit glatter Oberfläche (Abb.1). Der Lymphknotenstatus war unauffällig. Begleiterkrankungen wurden nicht festgestellt.

\section{Therapie und Verlauf}

Der Tumor wurde in Lokalanästhesie weit im Gesunden mit einer Verdachtsdiagnose „trichilemmale Zyste“ exzidiert. Der postoperative Verlauf war regelrecht. Die Patientin ist seit 7 Jahren erscheinungs- und beschwerdefrei geblieben.

Institutsangaben

${ }^{1}$ Hautklinik, Klinikum Heilbronn (Direktor: Prof. Dr. med. Dj. Djawari)

${ }^{2}$ Institut für Pathologie, Klinikum Heilbronn (Direktor: Prof. Dr. med. H. J. Rumpelt) Akademisches

Lehrkrankenhaus der Universität Heidelberg

Korrespondenzadresse

Prof. Dr. med. Dj. Djawari · Hautklinik am Gesundbrunnen · 74064 Heilbronn

Bibliografie

Akt Dermatol 2001; 27: 414-416 @ G Georg Thieme Verlag Stuttgart · New York · ISSN 0340-2541 


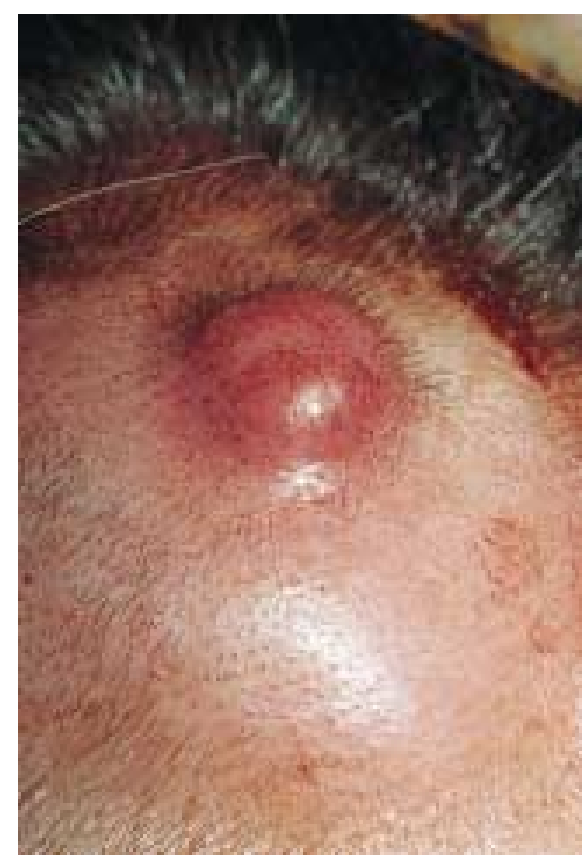

Abb. 1 Solitärer Herd einer Angiolymphoiden Hyperplasie mit Eosinophilie (AHE) bei einer 49-jährigen Patientin am behaarten Kopf.

Histologie

Der in toto exzidierte Knoten zeigte histologisch eine irreguläre Proliferation von Blutgefäßen, mit unterschiedlicher Wanddicke und „geschwollenem“ Endothel. Wobei fokal große Endothelzellen mit eosinophilem Zytoplasma und vergrößerten pleomorphen Zellkernen imponierten. Das umgebende Stroma war kollagenreich, mit lockerer entzündlicher Infiltration durch Lymphozyten, Plasmazellen, schaumzellig transformierte Histiozyten und vermehrten eosinophilen Granulozyten.

\section{Diskussion}

Die Angiolymphoide Hyperplasie mit Eosinophilie (AHE) wurde 1969 erstmalig von Wells und Whimster beschrieben [13]. Bereits 1948 hatte jedoch Kimura auf ein ähnliches Krankheitsbild, das durch subkutane Tumoren gekennzeichnet ist, hingewiesen [6].

Wells und Whimster betrachteten die AHE und den Morbus Kimura als frühes bzw. spätes Stadium einer Krankheitsentität. Aktuell werden diese Erkrankungen jedoch als zwei verschiedene Krankheitsbilder angesehen [3,5,7].

Weiterhin wurde der Sachverhalt dadurch kompliziert, dass die AHE unter verschiedensten Bezeichnungen beschrieben wurde, so zum Beispiel atypisches Granuloma pyogenicum, pseudopyogenisches Granulom, papulöse Angioplasie, angioplastische lymphoide Hyperplasie, inflammatorisches arterio-venöses Hämangiom, epitheloides Hämangiom und histiozytoides Hämangiom $[4,15]$. Die Bezeichnung Angiolymphoide Hyperplasie mit Eosinophilie kennzeichnet die Erkrankung histologisch am besten und ist in der Literatur am weitesten verbreitet [2,12].

Die AHE ist eine seltene Erkrankung, die in jedem Lebensalter auftreten kann. Sie ist klinisch charakterisiert durch solitäre angiomatoide Knoten oder Papeln, vorzugsweise an Kopf, Nacken,
Hals sowie retroaurikulär. Weniger häufige Lokalisationen sind Stamm, Extremitäten und Genitale. Selten kommen disseminierte Formen auch im Vergleich zu solitären Herden vor. Die Einzeleffloreszenz ist nodulär, glatt, glänzend und haarlos.

Die Konsistenz ist weich bis derb. Falls multiple Knoten auftreten, können diese konfluieren bzw. aggregieren. Es handelt sich um eine symptomlose Erkrankung, kann jedoch auch zu Juckreiz oder Schmerzhaftigkeit führen.

Differenzialdiagnostisch kommen klinisch Granuloma pyogenicum, kapilläre Hämangiome, Angiosarkome, Kaposi-Sarkom, Hämangiosarkome, Trichilemmalzysten, Fibrome, neurogene Tumoren und Hautmetastasen interner Neoplasien infrage $[1,12,14]$. Die Klärung der Diagnose ist nur histologisch möglich [7].

Histologisch ist die AHE gekennzeichnet durch eine Kombination aus abnormen Gefäßproliferaten und gemischtzelliger Entzündungsreaktion. Beide Komponenten können innerhalb des Einzelfalles und zwischen verschiedenen Fällen stark variieren. Das Gefäßspektrum umfasst dickwandige und dünnwandige Gefäße variabler Größe (z.T. mit arteriovenöser Shuntfunktion) bis zu sinusoidalen Gefäßspalten zwischen Endothelaggregaten.

Die Endothelzellen sind typischerweise voluminös und großkernig. Mitosen sind selten. Kernatypien kommen nicht vor. Das Endothel ist meist einlagig, kann auch mehrlagig proliferiert sein [7]. Das entzündliche Infiltrat enthält in variabler Mischung Lymphozyten, Plasmazellen, eosinophile Granulozyten, Mastzellen und Histiozyten. Letztere können Schaumzellen und histiozytäre Riesenzellen bilden. Besonders im Randbereich der Läsion sind meist zahlreiche große Lymphfollikel mit großen Keimzentren vorhanden (Abb. 2 ).

Immunhistochemische Untersuchungen haben gezeigt, dass die Endothelzellen positiv auf Anti-Faktor-VIII und Anti-UEA-1 reagieren [5]. Ultrastrukturell zeigen die Endothelzellen so genannte „Weibel-Palade-Körperchen“, welche gespeichertem FaktorVIII-assoziierten Antigen entsprechen.

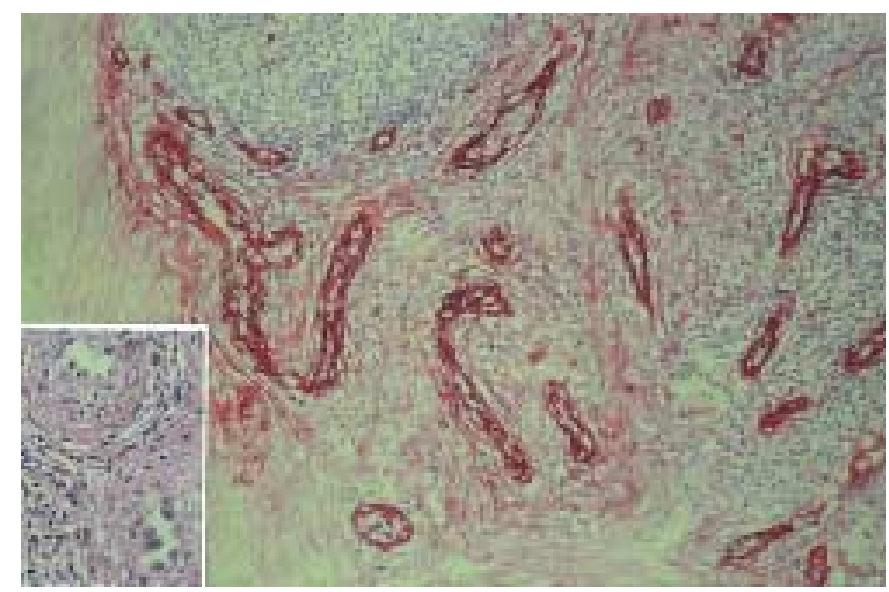

Abb. 2 Die Histologie der Angiolymphoiden Hyperplasie mit Eosinophilie unserer Patientin. Die zonale Gliederung der Läsion mit abnormen Gefäßproliferationen sowie intervaskulärem gemischten Entzündungsinfiltrat im Zentrum und Lymphfollikel in der Peripherie. Der abnorme schalenförmige Wandaufbau der Gefäße sowie die voluminösen Endothelzellen ohne Kernatypien sind typisch (CD 34 Färbung $\times 70$, Inset $\mathrm{HE} \times 560$ ). 
Tab. 1 Differenzierung zwischen der Angiolymphoiden Hyperplasie mit Eosinophilie (AHE) und Morbus Kimura

\begin{tabular}{|c|c|}
\hline $\mathrm{AHE}$ & M. Kimura \\
\hline $\begin{array}{l}\text { überall vorkommend } \\
\text { sporadisch }\end{array}$ & endemisch in Fernost \\
\hline $\begin{array}{l}\text { papulo-nodös } \\
\text { kutan-subkutan } \\
\text { (oberflächlich) }\end{array}$ & $\begin{array}{l}\text { tumorös } \\
\text { subkutan } \\
\text { (tief) }\end{array}$ \\
\hline $\begin{array}{l}\text { solitär } \\
\text { lokal begrenzte } \\
\text { vaskuläre Erkrankung }\end{array}$ & $\begin{array}{l}\text { multipel } \\
\text { lokal destruierende } \\
\text { chronisch-inflammatorische } \\
\text { Erkrankung mit Systembeteiligung } \\
\text { und LK-Befall }\end{array}$ \\
\hline$E>\Gamma$ & $\Gamma>E$ \\
\hline $\begin{array}{l}\text { Serum-IgE: normal } \\
\text { keine periphere Eosinophilie }\end{array}$ & $\begin{array}{l}\text { Serum-IgE: erhöht } \\
\text { periphere Eosinophilie }\end{array}$ \\
\hline $\begin{array}{l}\text { abnorme Gefäßproliferation mit } \\
\text { Endothelhyperplasie und } \\
\text { entzündlichem Hintergrund }\end{array}$ & $\begin{array}{l}\text { Gefäße normal bis gering proliferiert } \\
\text { vorwiegend entzündlicher Aspekt } \\
\text { mit Lymphfollikeln }\end{array}$ \\
\hline im Infiltrat T > B Lymphozyten & im Infiltrat B > T Lymphozyten \\
\hline
\end{tabular}

Die Ätiologie der AHE ist ungeklärt. In einigen Fällen wurde über ein vorheriges Trauma berichtet. Es wurden jedoch auch immunologische Ursachen diskutiert. Oft finden sich arteriovenöse Shunts, so dass es sich wahrscheinlich bei der AHE um einen reaktiven hyperplastischen Prozess, als Resultat eines möglichen Reparaturschadens eines arteriellen oder venösen Gefäßes, handelt [8]. Die Rezidivneigung könnte zum Beispiel durch posttherapeutisch verbliebene Shunts erklärt werden. Aus heutiger Sicht stellt die AHE kein neoplastisches Geschehen dar. Obwohl klinisch die differenzialdiagnostische Abgrenzung Schwierigkeiten bereitet, erfolgt die Diagnosefindung primär histologisch mit einem klar definierten Bild.

Der Morbus Kimura hingegen lässt sich sowohl klinisch als auch histopathologisch differenzieren [3-5]. Bei diesem liegt im Allgemeinen Lymphadenopathie, Bluteosinophilie und ggfs. eine Erhöhung des Serum-Gesamt-IgE vor. Histologisch zeigt der Morbus Kimura keine Gefäßanomalien, hingegen finden sich lymphoide Follikel mit reichlich eosinophilen Granulozyten [7]. Er wird als eine entzündliche Systemerkrankung unbekannter Ursache aufgefasst, während bei der AHE die Gefäßproliferation im Vordergrund steht (Tab.1).
Die unbehandelte AHE verläuft chronisch-progressiv, ohne Neigung zur Spontanremission. Therapeutisch ist aufgrund der Rezidivfreudigkeit eine In-toto-Exzision anzustreben [14]. Alternativ stehen Kryochirurgie, Lasertherapie, Radiotherapie sowie medikamentöse Behandlungen mit Isotretinoin, Pentoxyphillin und Kortikosteroiden zur Verfügung [9-11].

\section{Literatur}

${ }^{1}$ Bartralot R, Garcia-Patos V, Hueto J, Huguet P, Raspall G, Castells A. Angiolymphoid hyperplasia with eosinophilia affecting the oral mucosa: report of a case and a review of the literature. $\mathrm{Br} \mathrm{J}$ Dermatol 1996; 134: 744-748

2 Bunse T, Kuhn A, Groth W, Mahrle G. Therapeutisches Problem - Angiolymphoide Hyperplasie mit Eosinophilie. Hautarzt 1993; 44: 225-228

${ }^{3}$ Chun SI, Ji HG. Kimura's disease and angiolymphoid hyperplasia with eosinophilia: Clinical and histopathologic differences. J Am Acad Dermatol 1992; 27: $954-958$

${ }^{4}$ Gumbs MA, Pai NB, Saraiya RJ, Rubinstein J, Vythilingam L, Choi YJ. Kimura's disease: a case report and literature review. J Surg Oncol 1999; 70: $190-193$

${ }^{5}$ Helander SD, Peters MS, Kuo TT, Su WP. Kimura's disease and angiolymphoid hyperplasia with eosinophilia: new observations from immunohistochemical studies of lymphocyte markers, endothelial antigens an granulocyte proteins. J cut Pathol 1995; 22: 19-26

${ }^{6}$ Kimura T, Yoshimura S, Ishikawa E. On the unusual granulation combined with hyperplastic changes of lymphatic tissues. Trans Soc Pathol Jap 1948; 37: 179- 180

${ }^{7}$ Olsen TG, Helwig EB. Angiolymphoid hyperplasia with eosinophilia: A clinicopathologic study of 116 patients. J Am Acad Dermatol 1985; 12: $781-796$

${ }^{8}$ Onishi Y, Ohara K. Angiolymphoid hyperplasia with eosinophilia associated with arteriovenous malformation: a clinicopathological correlation with angiography and serial estimation of serum levels of renin, eosinophil cationic protein and interleukin 5. Br J Dermatol 1999; 140: $1153-1156$

9 Papadavid E, Krausz T, Chu AC, Walker NPJ. Angiolymphoid hyperplasia with eosinophilia - successfully treated with the flash-lamp pulsed-dye laser. Br J Dermatol 2000; 142: $192-194$

${ }^{10}$ Person JR. Angiolymphoid hyperplasia with eosinophilia may respond to pentoxifylline. J Am Acad Dermatol 1994; 31: 117- 118

${ }^{11}$ Rohrer TE, Allan AE. Angiolymphoid hyperplasia with eosinophilia successfully treated with a long-pulsed tunable dye laser. Dermatol Surg 2000; 26: $211-214$

${ }^{12}$ Schirren CG, Eckert F. Angiolymphoide Hyperplasie mit Eosinophilen. Hautarzt 1991; 42: 107-111

${ }^{13}$ Wells GC, Whimster IW. Subcutaneous angiolymphoid hyperplasia with eosinophilia. Br J Dermatol 1969; 81: 1-15

${ }^{14}$ Wolff $\mathrm{HH}$. Angiolymphoide Hyperplasie mit Eosinophilie. Z Hautkr 1986; 61: 767-770

${ }^{15}$ Wustrow A, Mahrle G. Angiolymphoide Hyperplasie mit Eosinophilie. Ein Spektrum von Morbus Kimura bis zum atypischen Granuloma pyogenicum. Z Hautkr 1987; 62: 613-620 